\title{
Azela-Ascoli Theorem and Its Applications
}

\author{
Emmanuel Akweittey*1, Gabriel O. Fosu ${ }^{1}$, Mubarack \\ Ahmed $^{2}$, Yarhands Dissou Arthur ${ }^{3}$ \\ ${ }^{1}$ Department of Mathematics, Presbyterian University College, Abetif, Ghana. \\ ${ }^{2}$ Department of Information Technology, Garden City University College, Kumasi, Ghana. \\ ${ }^{3}$ Department of Mathematics Education, University of education, Winneba, Kumasi - \\ Campus, Ghana.
}

\begin{abstract}
In this study we looked at the theorm Azela-Ascoli and its application to functional analysis, ordinary differential equations and complex analysis. This theorem simplifies the checking of compactness for subsets of spaces of continuous functions in much the same way the Heine-Borel theorem does for subsets of $R^{n}$.
\end{abstract}

Keywords: Azela-Ascoli theorem, Application, compactness and continuous functions.

\section{Introduction}

The problem of proving the compactness of various subsets of a given metric space is encountered quite frequently in analysis[1]. The Azela-Ascoli theorem is a fundamental theorem of analysis that allows us to determine if a subset of a space of continuous functions is compact. Although there are a number of ways to determine if a subset of a metric is compact, the Arzela-Ascoli theorem is often easier to apply than general conditions of completeness and total boundedness when working in spaces of functions[1]. This theorem simplifies checking of compactness for subsets of spaces of continuous functions in much the same way the Heine-Borel theorem does for subsets of $R^{n}$ [2]. It also has several applications in other branches of mathematics especially in ordinary differential equations and other branches of science and engineering.

\section{Statement and proof of the theorem}

If $X$ is a compact metric space, then a closed subspace of $C(X, \mathbb{R})$ or $C(X, \mathbb{C})$ is compact $\Leftrightarrow$ it is bounded and equicontinuous [3].

Proof

Let $d$ be the metric on $X$ and let $F$ be a closed of $C(X, \mathbb{R})$ or $C(X, \mathbb{C})$. We first assume that $F$ is compact, and we prove that it is bounded and equicontinuous. Then $F$ is bounded. 
We prove that $F$ is equicontinuous as follows:

Let $\epsilon>0$ be given. Since $F$ is compact and therefore totally bounded, we can find an $\left(\frac{\epsilon}{3}\right)-$ net $f_{1}, f_{2}, . ., f_{n} \in F$.

Each $f_{k}$ is uniformly continuous and so for each $k=1,2,3, . ., n$, there exist $\delta_{k}>0$ such that

$$
d\left(x, x^{\prime}\right)<\delta_{k} \Rightarrow\left|f_{k}(x)-f_{k}\left(x^{\prime}\right)\right|<\frac{\epsilon}{3}
$$

We now define $\delta$ to be the smallest of the numbers $\delta_{1}, \delta_{2}, \ldots, \delta_{n}$. If $f$ is any function in $F$ and $f_{k}$ is chosen so that $\left\|f-f_{k}\right\|<\frac{\epsilon}{3}$. Then

$d\left(x, x^{\prime}\right)<\delta \Rightarrow\left|f(x)-f\left(x^{\prime}\right)\right| \leq\left|f(x)-f_{k}(x)\right|+\left|f_{k}(x)-f_{k}\left(x^{\prime}\right)\right|+\left|f_{k}\left(x^{\prime}\right)-f\left(x^{\prime}\right)\right|<\frac{\delta}{3}+\frac{\delta}{3}+\frac{\delta}{3}=3$

This shows that $F$ is equicontinuous.

We now assume that $F$ is bounded and equicontinuous and we demonstrate that it is compact by showing that every sequence in it has a convergent subspace. Since $F$ is closed and therefore complete, it suffices to show that every sequence in it has a Cauchy sequence.

$X$ has a countable dense subset.

Let the points of this subset be arrange in a sequence $x_{i}=x_{1}, x_{2}, . ., x_{i}, \ldots$, where we start with the subscript 2 for reasons which will become clear below.

Now let $S_{1}=\left\{f_{11}, f_{12}, \ldots\right\}$ be an arbitrary sequence in $F$.

Our hypothesis that $F$ is bounded means that a there exist a real number $k$ such that $\|f\| \leq k$ for every $f$ in $F$ and every $x$ in $X$.

Consider the sequence of numbers $f_{i j}\left(x_{2}\right), j=1,2, \ldots$ and observe that since this sequence is bounded, it has a convergent subsequence.

Let $S_{2}=\left\{f_{21}, f_{22}, f_{23}, \ldots\right\}$ be a sequence of $S_{1}$ such that $f_{2 j}\left(x_{2}\right)$ converge.

We next consider the sequence of numbers $f_{3 j}\left(x_{3}\right)$ and in the same way we let $S_{3}=$ $\left\{f_{31}, f_{32}, f_{33}, \ldots\right\}$ be a subsequence of $S_{2}$ such that $f_{3 j}\left(x_{3}\right)$ converge.

If we continue this process, we get an array of sequence of the form

$S_{1}=\left\{f_{11}, f_{12}, f_{13}, \ldots\right\}$,

$S_{2}=\left\{f_{21}, f_{22}, f_{23}, \ldots\right\}$

$S_{3}=\left\{f_{31}, f_{32}, f_{33}, \ldots\right\}$

in which each sequence is a subsequence of the one directly above it and for each $i$ the sequence $S_{i}=\left\{f_{i 1}, f_{i 2}, f_{i 3}, \ldots\right\}$ has the property that $f_{i j}\left(x_{i}\right)$ is a convergent sequence of numbers.

If we define $f_{1}, f_{2}, f_{3}, \ldots$ by $f_{1}=f_{11}, f_{2}=f_{22}, \ldots \ldots$, then the sequence $S=\left\{f_{1}, f_{2}, f_{3}, \ldots\right\}$ is the diagonal subsequence of $S_{1}$. It is clear from this construction that for each point $x_{i}$ in our dense subset of $X$, the sequence $f_{n}\left(x_{i}\right)$ is a convergent sequence of numbers.

It remains only to show that $S$ as a sequence of functions in $C(X, \mathbb{R})$ or $C(X, \mathbb{C})$ is a Cauchy sequence.

Let $\epsilon>0$ be given. Since $F$ is equicontinuous, there exist $\delta>0$ such that $d\left(x, x^{\prime}\right)<\delta \Rightarrow$ $\left|f_{n}(x)-f_{n}\left(x^{\prime}\right)\right|<\frac{\epsilon}{3}$ for all functions in $f_{n}$ in $S$.

We now form the open spheres $S_{\delta}\left(x_{i}\right)$ with radius $\delta$ centered on each of the $x_{i}^{\prime}$ s. Since the $x_{i}^{\prime}$ s are dense, these open spheres form an open cover of $X$ and since $X$ is compact, $X=\cup_{i=2}^{i_{0}} S_{\delta}\left(x_{i}\right)$ for some $i_{0}$. 
It is easy to see that there exist a positive integer $n_{0}$ such that $m, n \geq n_{0}\left|f_{m}\left(x_{i}\right)-f_{n}\left(x_{i}\right)\right|<\frac{\delta}{3}$ for all the points $x_{2}, x_{3}, . ., x_{i_{0}}$.

Our proof is completed by the remark that if $x$ is an arbitrary point in $X$, then an $i$ can be found in the set $\left\{2,3,4, . ., i_{0}\right\}$ such that $d\left(x, x^{\prime}\right)<\delta$ and that therefore

$m, n \geq n_{0}\left|f_{m}(n)-f_{n}(n)\right| \leq\left|f_{m}(n)-f_{n}\left(x_{i}\right)\right|+\left|f_{m}\left(x_{i}\right)-f_{n}\left(x_{i}\right)\right|+\left|f_{n}\left(x_{i}\right)-f_{n}(x)\right|<\frac{\epsilon}{3}+\frac{\epsilon}{3}+\frac{\epsilon}{3}=3$

$[3]$.

\subsection{Application of the Theorem}

Here we examine one application each to functional analysis, ordinary differential equation and complex analysis.

\subsection{Application to Functional Analysis}

For $f \in C[0,1]$, let $(T f)(x)=\int_{0}^{x} f(t) d t$. Then $T f \in C[0,1]$, so $T$ is a linear map from $C[0,1]$ to itself.

Let $F=\left\{T f: f \in C[0,1],\|f\|_{\infty} \leq 1\right\}$. We would like to see whether $F$ is equicontinuous. $|(T f)(x)-(T f)(y)|=\left|\int_{x}^{y} f(t) d t\right| \leq|x-y|$.

Hence $F$ is an equicontinuous family. Also $|(T f)(x)| \leq x \leq 1$. This implies that $F$ is bounded. Hence $F$ is compact[4].

\subsection{Application to Ordinary Differential Equation (Peano's Theorem)}

Let $f$ be a continuous function from a neighbourhood $U$ of $\left(0, x_{0}\right) \in R \times R^{n}$ to $R^{n}$. Then there exist an $\epsilon>0$ such that the initial-value problem $\frac{d x}{d t}=f(t, x(t)), x(0)=x_{0}$ has a solution $x$ on $[0, \epsilon][1]$.

\section{Proof}

Without loss of generality, assume that $U$ is of the form $[-\delta, \delta] \times B\left[x_{0}, r\right]$. Let $M$ be a bound for $f$ on $U$. Let $\epsilon=\min \left\{\delta, \frac{r}{m}\right\}$.

Define a sequence $\left(x_{n}\right)$ on $[0, \epsilon]$ as follows $x_{n}(t)= \begin{cases}x_{o} & t \in\left[0, \frac{\epsilon}{n}\right] \\ x_{0}+\int_{0}^{t}-\frac{\epsilon}{n} f\left(s, x_{n}(s)\right) & t \in\left(\frac{\epsilon}{n}, \epsilon\right]\end{cases}$

Observe that these formulae determine the function $x_{n}$ on $[0, \epsilon]$ since its values on $\left(\frac{k \epsilon}{n}, \frac{(k+1) \epsilon}{n}\right]$ are determine by its values on $\left[0, \frac{k \epsilon}{n}\right]$ for $1 \leq k \leq n-1$ and its values on $\left[0, \frac{\epsilon}{n}\right]$ are given. The family $\left\{x_{n}\right\}$ is equicontinuous on $[0, \epsilon]$.

Let $\left(x_{n_{k}}\right)$ converges to $x$. Then $x$ satisfies an integral equation which is equivalent to the given differential equation on $[0, \epsilon]$. 


\subsection{Application to Complex Analysis}

Let $U \epsilon C$, the complex space be open.

Let $\left\{f_{n}\right\}$ be a sequence of holomorphic functions on $U$. Assume that for each compact subset $K$ of $U$ there is a $M_{k}$ such that $\left|f_{n}(z)\right| \leq M_{k}$ for $z \in K$ and $n \in N$. Then there is a subsequence which converges to a holomorphic function on compact subset of $U$.

Hint: Use Cauchy integral formula to obtain equicontinuity. This result is used in proving Riemann Mapping Theory in Complex Analysis.

\subsection{Conclusion}

The Arzela-Ascoli theorem allows us to study compact sets in some function spaces. This theorem simplifies checking of compactness for subsets of spaces of continuous functions in much the same way the Heine-Borel theorem does for subsets of $R^{n}$.

Moreover, a lot of the topological spaces used in Real Analysis, Complex Analysis and Functional Analysis are spaces of functions. Azela-Ascoli theorem allows us to study the properties of these spaces of functions.

\section{References}

[1] A.N. Kolmogorov S.V. Fomin, Silverman Richard A.. Introductory Real Analysis. Dover Publications 1975.

[2] Rudin Walter. Functional Analysis. McGraw-Hill 1991.

[3] Simmons George F.. Introduction to topology and modern analysis. Krieger Publishing Company 2003.

[4] Gosffman Casper, Perdrick George. First Course in Functional Analysis. Prentice Hall; 1st edition 1965. 